\title{
Existence of mild solutions for fractional evolution equations with nonlocal conditions
}

He Yang*

\section{"Correspondence:} yanghe256@163.com

Department of Mathematics, Northwest Normal University, Lanzhou, 730070, People's Republic of China

\begin{abstract}
This paper deals with the existence and uniqueness of mild solutions for a class of fractional evolution equations with nonlocal initial conditions. We present some local growth conditions on a nonlinear part and a nonlocal term to guarantee the existence theorems. An example is given to illustrate the applicability of our results.
\end{abstract} MSC: $34 \mathrm{~A} 12 ; 35 \mathrm{~F} 25$

Keywords: fractional evolution equations; nonlocal initial conditions; existence; uniqueness

\section{Introduction}

The differential equations involving fractional derivatives in time have recently been proved to be valuable tools in the modeling of many phenomena in various fields of engineering and science. Indeed, we can find numerous applications in electrochemistry, control, porous media, electromagnetic processing etc. (see [1-5]). Hence, the research on fractional differential equations has become an object of extensive study during recent years; see [6-11] and references therein.

On the other hand, the nonlocal initial condition, in many cases, has much better effect in applications than the traditional initial condition. As remarked by Byszewski and Lakshmikantham (see $[12,13])$, the nonlocal initial value problems can be more useful than the standard initial value problems to describe many physical phenomena.

Let $X$ be a Banach space with norm $\|\cdot\|$, and let $T>0$ be a constant. Consider the existence and uniqueness of mild solutions of fractional evolution equation with nonlocal condition in the form

$$
\left\{\begin{array}{l}
D^{q} u(t)+A u(t)=f(t, u(t))+\int_{0}^{t} K(t, s) h(s, u(s)) d s, \quad t \in J=[0, T], \\
u(0)+g(u)=x_{0},
\end{array}\right.
$$

where $D^{q}$ is the Caputo fractional derivative of order $q \in(0,1)$, the linear operator $-A$ is the infinitesimal generator of an analytic semigroup $\{S(t)\}_{t \geq 0}$ in $X$, the functions $f, h$ and $g$ will be specified later. $K \in C\left(\Delta, \mathbb{R}^{+}\right)$, where $\Delta=\{(t, s) \mid 0 \leq s \leq t \leq T\}, \mathbb{R}^{+}=[0,+\infty)$. Throughout this paper, we always assume that $K^{*}=\max _{(t, s) \in \Delta} K(t, s)$.

In some existing articles, the fractional differential equations with nonlocal initial conditions were treated under the hypothesis that the nonlocal term is completely continuous or global Lipschitz continuous. It is obvious that these conditions are not easy to verify in

(c) 2012 Yang; licensee Springer. This is an Open Access article distributed under the terms of the Creative Commons Attribution License (http://creativecommons.org/licenses/by/2.0), which permits unrestricted use, distribution, and reproduction in any medium, provided the original work is properly cited. 
many cases. To make the things more applicable, in [6] the authors studied the existence and uniqueness of mild solutions of Eq. (1) under the case $K(t, s) \equiv 0$. In their main results, they did not assume the complete continuity of the nonlocal term, but they needed the following assumptions:

(F1) there exist a constant $q_{1} \in[0, q)$ and $m \in L^{\frac{1}{q_{1}}}\left(J, \mathbb{R}^{+}\right)$such that $\|f(t, x)\| \leq m(t)$ for all $x \in X$ and almost all $t \in J$;

(F2) there exists a constant $L>0$ such that $\|g(u)-g(v)\| \leq L\|u-v\|$ for $u, v \in C(J, X)$; and some other conditions.

In this paper, we will improve the conditions (F1) and (F2). We only assume that $f$ and $h$ satisfy local growth conditions (see assumption $\left(\mathrm{H}_{3}\right)$ ) and $g$ is local Lipschitz continuous (see assumption $\left(\mathrm{H}_{4}\right)$ ). We will carry out our investigation in the Banach space $X_{\alpha}:=\left(D\left(A^{\alpha}\right),\|\cdot\|_{\alpha}\right), 0<\alpha<1$, where $D\left(A^{\alpha}\right)$ is the domain of the fractional power of $A$. Finally, an example is given to illustrate the applicability of our main results. We can see that the main results in [6] cannot be applied to our example.

The rest of this paper is organized as follows. In Section 2, some preliminaries are given on the fractional power of the generator of an analytic semigroup and on the mild solutions of Eq. (1). In Section 3, we study the existence and uniqueness of mild solutions of Eq. (1). In Section 4, we give an example to illustrate the applicability of our results.

\section{Preliminaries}

Let $X$ be a Banach space with norm $\|\cdot\|$, and let $-A: D(A) \subset X \rightarrow X$ be the infinitesimal generator of an analytic semigroup $S(t)(t \geq 0)$ of a uniformly bounded linear operator in $X$, that is, there exists $M \geq 1$ such that $\|S(t)\| \leq M$ for all $t \geq 0$. Without loss of generality, let $0 \in \rho(A)$. Then for any $\alpha>0$, we can define $A^{-\alpha}$ by

$$
A^{-\alpha}=\frac{1}{\Gamma(\alpha)} \int_{0}^{\infty} t^{\alpha-1} S(t) d t
$$

$A^{-\alpha} \in \mathcal{L}(X)$ is injective, and $A^{\alpha}$ can be defined by $A^{\alpha}=\left(A^{-\alpha}\right)^{-1}$ with the domain $D\left(A^{\alpha}\right)=$ $A^{-\alpha}(X)$. For $\alpha=0$, let $A^{\alpha}=I$.

Lemma 1 ([14]) $A^{\alpha}$ has the following properties:

(i) $D\left(A^{\alpha}\right)$ is a Banach space with the norm $\|x\|_{\alpha}:=\left\|A^{\alpha} x\right\|$ for $x \in D\left(A^{\alpha}\right)$;

(ii) $S(t): X \rightarrow X_{\alpha}$ for each $t>0$;

(iii) $A^{\alpha} S(t) x=S(t) A^{\alpha} x$ for each $x \in D\left(A^{\alpha}\right)$ and $t \geq 0$;

(iv) $A^{-\alpha}$ is a bounded linear operator on $X$ with $D\left(A^{\alpha}\right)=\operatorname{Im}\left(A^{-\alpha}\right)$;

(v) If $0<\alpha \leq \beta$, then $D\left(A^{\beta}\right) \hookrightarrow D\left(A^{\alpha}\right)$.

Let $X_{\alpha}$ be the Banach space of $D\left(A^{\alpha}\right)$ endowed with the norm $\|\cdot\|_{\alpha}$. Denote by $C\left(J, X_{\alpha}\right)$ the Banach space of all continuous functions from $J$ into $X_{\alpha}$ with the supnorm given by $\|u\|_{C}=\sup _{t \in J}\|u(t)\|_{\alpha}$ for $u \in C\left(J, X_{\alpha}\right)$. From Lemma 1(iv), since $A^{-\alpha}$ is a bounded linear operator for $\alpha>0$, we denote by $C_{\alpha}$ the operator norm of $A^{-\alpha}$ in $X$, that is, $C_{\alpha}:=\left\|A^{-\alpha}\right\|$. For any $t \geq 0$, denote by $S_{\alpha}(t)$ the restriction of $S(t)$ to $X_{\alpha}$. From Lemma 1(ii) and (iii), for any $x \in X_{\alpha}$, we have

$$
\|S(t) x\|_{\alpha}=\left\|A^{\alpha} \cdot S(t) x\right\|=\left\|S(t) \cdot A^{\alpha} x\right\| \leq\|S(t)\| \cdot\left\|A^{\alpha} x\right\|=\|S(t)\| \cdot\|x\|_{\alpha},
$$


and

$$
\|S(t) x-x\|_{\alpha}=\left\|A^{\alpha} \cdot S(t) x-A^{\alpha} x\right\|=\left\|S(t) \cdot A^{\alpha} x-A^{\alpha} x\right\| \rightarrow 0
$$

as $t \rightarrow 0$. Therefore, $S(t)(t \geq 0)$ is a strongly continuous semigroup in $X_{\alpha}$, and $\left\|S_{\alpha}(t)\right\|_{\alpha} \leq$ $\|S(t)\|$ for all $t \geq 0$. To prove our main results, the following lemma is needed.

Lemma 2 ([15]) If $S(t)(t \geq 0)$ is a compact semigroup in $X$, then $S_{\alpha}(t)(t \geq 0)$ is an immediately compact semigroup in $X_{\alpha}$, and hence it is immediately norm-continuous.

For $x \in X$, define two families $\{U(t)\}_{t \geq 0}$ and $\{V(t)\}_{t \geq 0}$ of operators by

$$
U(t) x=\int_{0}^{\infty} \eta_{q}(\theta) S\left(t^{q} \theta\right) x d \theta, \quad V(t) x=q \int_{0}^{\infty} \theta \eta_{q}(\theta) S\left(t^{q} \theta\right) x d \theta, \quad 0<q<1,
$$

where

$$
\begin{aligned}
& \eta_{q}(\theta)=\frac{1}{q} \theta^{-1-\frac{1}{q}} \rho_{q}\left(\theta^{-\frac{1}{q}}\right), \\
& \rho_{q}(\theta)=\frac{1}{\pi} \sum_{n=1}^{\infty}(-1)^{n-1} \theta^{-q n-1} \frac{\Gamma(n q+1)}{n !} \sin (n \pi q), \quad \theta \in(0, \infty),
\end{aligned}
$$

where $\eta_{q}$ is the probability density function defined on $(0, \infty)$, which has properties $\eta_{q}(\theta) \geq 0$ for all $\theta \in(0, \infty)$ and

$$
\int_{0}^{\infty} \eta_{q}(\theta) d \theta=1, \quad \int_{0}^{\infty} \theta \eta_{q}(\theta) d \theta=\frac{1}{\Gamma(1+q)} .
$$

The following lemma follows from the results in $[6-8,10]$.

Lemma 3 The following properties are valid:

(i) For fixed $t \geq 0$ and any $x \in X_{\alpha}$, we have

$$
\|U(t) x\|_{\alpha} \leq M\|x\|_{\alpha}, \quad\|V(t) x\|_{\alpha} \leq \frac{q M}{\Gamma(1+q)}\|x\|_{\alpha}=\frac{M}{\Gamma(q)}\|x\|_{\alpha} .
$$

(ii) The operators $U(t)$ and $V(t)$ are strongly continuous for all $t \geq 0$.

(iii) If $S(t)(t \geq 0)$ is a compact semigroup in $X$, then $U(t)$ and $V(t)$ are norm-continuous in $X$ for $t>0$.

(iv) If $S(t)(t \geq 0)$ is a compact semigroup in $X$, then $U(t)$ and $V(t)$ are compact operators in $X$ for $t>0$.

In this paper, we adopt the following definition of a mild solution of Eq. (1).

Definition 1 By a mild solution of Eq. (1), we mean a function $u \in C\left(J, X_{\alpha}\right)$ satisfying

$$
u(t)=U(t)\left(x_{0}-g(u)\right)+\int_{0}^{t}(t-s)^{q-1} V(t-s)\left[f(s, u(s))+\int_{0}^{s} K(s, \tau) h(\tau, u(\tau)) d \tau\right] d s
$$

for all $t \in J$. 
To prove our main results, we also need the following two lemmas.

Lemma 4 A measurable function $H:[0, T] \rightarrow X$ is Bochner integrable if $|H|$ is Lebesgue integrable.

Lemma 5 (Krasnoselskii's fixed point theorem) Let $X$ be a Banach space, let $B$ be a bounded closed and convex subset of $X$ and let $Q_{1}$ and $Q_{2}$ be mappings from $B$ into $X$ such that $Q_{1} x+Q_{2} y \in B$ for every pair $x, y \in B$. If $Q_{1}$ is a contraction and $Q_{2}$ is completely continuous, then the operator equation $Q_{1} x+Q_{2} x=x$ has a solution on $B$.

Lemmas 4 and 5 , which can be found in many books, are classical.

The following are the basic assumptions of this paper.

$\left(\mathrm{H}_{1}\right) S(t)(t \geq 0)$ is a compact operator semigroup in $X$.

$\left(\mathrm{H}_{2}\right)$ There exists a constant $\beta \in[\alpha, 1]$ such that the functions $f, h: J \times X_{\alpha} \rightarrow X_{\beta}$ satisfy the following conditions:

(i) For each $x \in X_{\alpha}$, the functions $f(\cdot, x), h(\cdot, x)$ are measurable.

(ii) For each $t \in J$, the functions $f(t, \cdot), h(t, \cdot)$ are continuous.

$\left(\mathrm{H}_{3}\right)$ For $t \in J$ and $r>0$, there exist positive functions $\varphi_{r}$ satisfying $\frac{\varphi_{r} \cdot(\cdot)}{(t-\cdot)^{1-q}} \in L^{1}\left([0, t], \mathbb{R}^{+}\right)$ and $\phi_{r} \in L^{1}\left(J, \mathbb{R}^{+}\right)$such that

$$
\sup _{\|x\|_{\alpha} \leq r}\|f(t, x)\|_{\beta} \leq \varphi_{r}(t), \quad \sup _{\|x\|_{\alpha} \leq r}\|h(t, x)\|_{\beta} \leq \phi_{r}(t)
$$

and there are positive constants $\sigma_{1}$ and $\sigma_{2}$ such that

$$
\liminf _{r \rightarrow+\infty} \frac{1}{r} \int_{0}^{t} \frac{\varphi_{r}(s)}{(t-s)^{1-q}} d s \leq \sigma_{1}<+\infty, \quad \liminf _{r \rightarrow+\infty} \frac{1}{r} \int_{0}^{t} \frac{\int_{0}^{s} \phi_{r}(\tau) d \tau}{(t-s)^{1-q}} d s \leq \sigma_{2}<+\infty
$$

$\left(\mathrm{H}_{4}\right) g: C\left(J, X_{\alpha}\right) \rightarrow X_{\alpha}$ and for $r>0$, there exists a positive constant $L$ such that

$$
\|g(u)-g(v)\|_{\alpha} \leq L\|u-v\|_{C}
$$

for all $u, v \in B_{r}:=\left\{u \in C\left(J, X_{\alpha}\right):\|u(t)\|_{\alpha} \leq r, t \in J\right\}$.

\section{Main results}

In this section, we introduce the existence and uniqueness theorems of mild solutions of Eq. (1). The discussions are based on fractional calculus and Krasnoselskii's fixed point theorem. Our main results are as follows.

Theorem 1 Suppose that the assumptions $\left(\mathrm{H}_{1}\right)-\left(\mathrm{H}_{4}\right)$ hold. If $x_{0} \in X_{\alpha}$ and the following inequality holds:

$$
M L+\frac{M C_{\beta-\alpha}}{\Gamma(q)}\left(\sigma_{1}+K^{\prime \prime} \sigma_{2}\right)<1
$$

then Eq. (1) has at least one mild solution on J. 
Proof Define two operators $Q_{1}$ and $Q_{2}$ as follows:

$$
\begin{aligned}
& \left(Q_{1} u\right)(t)=U(t)\left(x_{0}-g(u)\right), \quad t \in J, \\
& \left(Q_{2} u\right)(t)=\int_{0}^{t}(t-s)^{q-1} V(t-s)\left[f(s, u(s))+\int_{0}^{s} K(s, \tau) h(\tau, u(\tau)) d \tau\right] d s, \quad t \in J .
\end{aligned}
$$

Obviously, $u$ is a mild solution of Eq. (1) if and only if $u$ is a solution of the operator equation $u=Q_{1} u+Q_{2} u$ on $J$. To prove the operator equation $u=Q_{1} u+Q_{2} u$ has solutions, we first show that there is a positive number $r$ such that $Q_{1} u+Q_{2} v \subset B_{r}$ for every pair $u, v \in B_{r}$. If this were not the case, then for each $r>0$, there would exist $u_{r}, v_{r} \in B_{r}$ and $t_{r} \in J$ such that $\left\|\left(Q_{1} u_{r}\right)\left(t_{r}\right)+\left(Q_{2} v_{r}\right)\left(t_{r}\right)\right\|_{\alpha}>r$. Thus, from Lemma 3, $\left(\mathrm{H}_{3}\right)$ and $\left(\mathrm{H}_{4}\right)$, we have

$$
\begin{aligned}
r< & \left\|\left(Q_{1} u_{r}\right)\left(t_{r}\right)+\left(Q_{2} v_{r}\right)\left(t_{r}\right)\right\|_{\alpha} \leq\left\|U\left(t_{r}\right)\left(x_{0}-g\left(u_{r}\right)\right)\right\|_{\alpha} \\
& +\int_{0}^{t_{r}}\left(t_{r}-s\right)^{q-1}\left\|V\left(t_{r}-s\right)\left[f\left(s, v_{r}(s)\right)+\int_{0}^{s} K(s, \tau) h\left(\tau, v_{r}(\tau)\right) d \tau\right]\right\|_{\alpha} d s \\
\leq & M\left\|x_{0}-\left[g\left(u_{r}\right)-g(0)\right]-g(0)\right\|_{\alpha} \\
& +\frac{M}{\Gamma(q)} \int_{0}^{t_{r}}\left(t_{r}-s\right)^{q-1}\left\|A^{\alpha-\beta} \cdot A^{\beta}\left[f\left(s, v_{r}(s)\right)+\int_{0}^{s} K(s, \tau) h\left(\tau, v_{r}(\tau)\right) d \tau\right]\right\| d s \\
\leq & M\left\|x_{0}\right\|_{\alpha}+M L r+M\|g(0)\|_{\alpha} \\
& +\frac{M C_{\beta-\alpha}}{\Gamma(q)} \int_{0}^{t_{r}}\left(t_{r}-s\right)^{q-1}\left[\varphi_{r}(s)+K^{*} \int_{0}^{s} \phi_{r}(\tau) d \tau\right] d s .
\end{aligned}
$$

Dividing on both sides by $r$ and taking the lower limit as $r \rightarrow+\infty$, we have

$$
M L+\frac{M C_{\beta-\alpha}}{\Gamma(q)}\left(\sigma_{1}+K^{\prime \prime} \sigma_{2}\right) \geq 1
$$

which contradicts (3). Hence, for some $r>0, Q_{1} u+Q_{2} v \subset B_{r}$ for every pair $u, v \in B_{r}$.

The next proof will be given in two steps.

Step 1. $Q_{1}$ is a contraction on $B_{r}$.

For any $u, v \in B_{r}$ and $t \in J$, according to Lemma 3 and assumption $\left(\mathrm{H}_{4}\right)$, we have

$$
\left\|\left(Q_{1} u\right)(t)-\left(Q_{1} v\right)(t)\right\|_{\alpha}=\|U(t)[g(v)-g(u)]\|_{\alpha} \leq M\|g(u)-g(v)\|_{\alpha} \leq M L\|u-v\|_{C},
$$

which implies that $\left\|Q_{1} u-Q_{1} v\right\|_{C} \leq M L\|u-v\|_{C}$. It follows from (3) that $M L<1$, hence $Q_{1}$ is a contraction on $B_{r}$.

Step 2. $Q_{2}$ is a completely continuous operator on $B_{r}$.

We first prove that $Q_{2}$ is continuous on $B_{r}$. Let $\left\{u_{n}\right\} \subset B_{r}$ with $u_{n} \rightarrow u \in B_{r}$ as $n \rightarrow \infty$. Then for any $t \in J, s \in[0, t]$, by assumption $\left(\mathrm{H}_{2}\right)$, we have

$$
f\left(s, u_{n}(s)\right) \rightarrow f(s, u(s)), \quad h\left(s, u_{n}(s)\right) \rightarrow h(s, u(s))
$$

as $n \rightarrow \infty$, and from assumption $\left(\mathrm{H}_{3}\right)$, we have

$$
\begin{aligned}
& \left\|f\left(s, u_{n}(s)\right)-f(s, u(s))\right\|_{\beta} \leq 2 \varphi_{r}(s), \quad\left\|h\left(s, u_{n}(s)\right)-h(s, u(s))\right\|_{\beta} \leq 2 \phi_{r}(s), \\
& \int_{0}^{s} K(s, \tau)\left\|h\left(\tau, u_{n}(\tau)\right)-h(\tau, u(\tau))\right\|_{\beta} d \tau \leq 2 K^{*}\left\|\phi_{r}\right\|_{L^{1}} .
\end{aligned}
$$


This together with the Lebesgue dominated convergence theorem gives that

$$
\begin{aligned}
& \left\|\left(Q_{2} u_{n}\right)(t)-\left(Q_{2} u\right)(t)\right\|_{\alpha} \\
& \leq \int_{0}^{t}(t-s)^{q-1}\left\|V(t-s) \cdot\left[f\left(s, u_{n}(s)\right)-f(s, u(s))\right]\right\|_{\alpha} d s \\
& \quad+\int_{0}^{t}(t-s)^{q-1}\left\|V(t-s) \cdot \int_{0}^{s} K(s, \tau)\left[h\left(\tau, u_{n}(\tau)\right)-h(\tau, u(\tau))\right] d \tau\right\|_{\alpha} d s \\
& \leq \frac{M}{\Gamma(q)} \int_{0}^{t}(t-s)^{q-1}\left\|A^{\alpha-\beta} \cdot A^{\beta}\left[f\left(s, u_{n}(s)\right)-f(s, u(s))\right]\right\| d s \\
& \quad+\frac{M}{\Gamma(q)} \int_{0}^{t}(t-s)^{q-1}\left\|A^{\alpha-\beta} \int_{0}^{s} K(s, \tau) \cdot A^{\beta}\left[h\left(\tau, u_{n}(\tau)\right)-h(\tau, u(\tau))\right] d \tau\right\| d s \\
& \leq \frac{M C_{\beta-\alpha}}{\Gamma(q)} \int_{0}^{t}(t-s)^{q-1}\left\|f\left(s, u_{n}(s)\right)-f(s, u(s))\right\|_{\beta} d s \\
& \quad+\frac{M C_{\beta-\alpha}}{\Gamma(q)} \int_{0}^{t}(t-s)^{q-1} \int_{0}^{s} K(s, \tau)\left\|h\left(\tau, u_{n}(\tau)\right)-h(\tau, u(\tau))\right\|_{\beta} d \tau d s \rightarrow 0
\end{aligned}
$$

as $n \rightarrow \infty$. Hence, $\lim _{n \rightarrow \infty}\left\|Q_{2} u_{n}-Q_{2} u\right\|_{C}=0$. This means that $Q_{2}$ is continuous on $B_{r}$.

Next, we will show that the set $\left\{Q_{2} u: u \in B_{r}\right\}$ is relatively compact. It suffices to show that the family of functions $\left\{Q_{2} u: u \in B_{r}\right\}$ is uniformly bounded and equicontinuous, and for any $t \in J$, the set $\left\{\left(Q_{2} u\right)(t): u \in B_{r}\right\}$ is relatively compact.

For any $u \in B_{r}$, we have $\left\|Q_{2} u\right\|_{C} \leq r$ for some $r>0$, which means that $\left\{Q_{2} u: u \in B_{r}\right\}$ is uniformly bounded. In what follows, we show that $\left\{Q_{2} u: u \in B_{r}\right\}$ is a family of equicontinuous functions. For $t \in[0, T)$, we have

$$
\left\|\left(Q_{2} u\right)(t)-\left(Q_{2} u\right)(0)\right\|_{\alpha} \leq \frac{M C_{\beta-\alpha}}{\Gamma(q)} \int_{0}^{t}(t-s)^{q-1}\left[\varphi_{r}(s)+K^{*} \int_{0}^{s} \phi_{r}(\tau) d \tau\right] d s .
$$

Hence, it is only necessary to consider $t>0$. For $0<t_{1}<t_{2} \leq T$, from Lemma 3 and assumption $\left(\mathrm{H}_{3}\right)$, we have

$$
\begin{aligned}
& \left\|\left(Q_{2} u\right)\left(t_{2}\right)-\left(Q_{2} u\right)\left(t_{1}\right)\right\|_{\alpha} \\
& \leq\left\|\int_{0}^{t_{1}}\left(t_{2}-s\right)^{q-1}\left[V\left(t_{2}-s\right)-V\left(t_{1}-s\right)\right] \cdot\left[f(s, u(s))+\int_{0}^{s} K(s, \tau) h(\tau, u(\tau)) d \tau\right] d s\right\|_{\alpha} \\
& \quad+\| \int_{0}^{t_{1}}\left[\left(t_{2}-s\right)^{q-1}-\left(t_{1}-s\right)^{q-1}\right] V\left(t_{1}-s\right) \\
& \quad \times\left[f(s, u(s))+\int_{0}^{s} K(s, \tau) h(\tau, u(\tau)) d \tau\right] d s \|_{\alpha} \\
& \quad+\left\|\int_{t_{1}}^{t_{2}}\left(t_{2}-s\right)^{q-1} V\left(t_{2}-s\right) \cdot\left[f(s, u(s))+\int_{0}^{s} K(s, \tau) h(\tau, u(\tau)) d \tau\right] d s\right\|_{\alpha} \\
& \leq \int_{0}^{t_{1}}\left(t_{2}-s\right)^{q-1} \| A^{\alpha-\beta}\left[V\left(t_{2}-s\right)-V\left(t_{1}-s\right)\right] \\
& \quad \times A^{\beta}\left[f(s, u(s))+\int_{0}^{s} K(s, \tau) h(\tau, u(\tau)) d \tau\right] \| d s \\
& \quad+\frac{M}{\Gamma(q)} \int_{0}^{t_{1}}\left|\left(t_{2}-s\right)^{q-1}-\left(t_{1}-s\right)^{q-1}\right|
\end{aligned}
$$




$$
\begin{aligned}
& \times\left\|A^{\alpha-\beta} \cdot A^{\beta}\left[f(s, u(s))+\int_{0}^{s} K(s, \tau) h(\tau, u(\tau)) d \tau\right]\right\| d s \\
& +\frac{M}{\Gamma(q)} \int_{t_{1}}^{t_{2}}\left(t_{2}-s\right)^{q-1}\left\|A^{\alpha-\beta} \cdot A^{\beta}\left[f(s, u(s))+\int_{0}^{s} K(s, \tau) h(\tau, u(\tau)) d \tau\right]\right\| d s \\
\leq & C_{\beta-\alpha} \int_{0}^{t_{1}}\left(t_{2}-s\right)^{q-1}\left\|V\left(t_{2}-s\right)-V\left(t_{1}-s\right)\right\| \cdot\left[\varphi_{r}(s)+K^{*} \int_{0}^{s} \phi_{r}(\tau) d \tau\right] d s \\
& +\frac{M C_{\beta-\alpha}}{\Gamma(q)} \int_{0}^{t_{1}}\left|\left(t_{2}-s\right)^{q-1}-\left(t_{1}-s\right)^{q-1}\right| \cdot\left[\varphi_{r}(s)+K^{*} \int_{0}^{s} \phi_{r}(\tau) d \tau\right] d s \\
& +\frac{M C_{\beta-\alpha}}{\Gamma(q)} \int_{t_{1}}^{t_{2}}\left(t_{2}-s\right)^{q-1} \cdot\left[\varphi_{r}(s)+K^{*} \int_{0}^{s} \phi_{r}(\tau) d \tau\right] d s \\
\triangleq & I_{1}+I_{2}+I_{3},
\end{aligned}
$$

where

$$
\begin{aligned}
& I_{1}=C_{\beta-\alpha} \int_{0}^{t_{1}}\left(t_{2}-s\right)^{q-1}\left\|V\left(t_{2}-s\right)-V\left(t_{1}-s\right)\right\| \cdot\left[\varphi_{r}(s)+K^{*} \int_{0}^{s} \phi_{r}(\tau) d \tau\right] d s, \\
& I_{2}=\frac{M C_{\beta-\alpha}}{\Gamma(q)} \int_{0}^{t_{1}}\left|\left(t_{2}-s\right)^{q-1}-\left(t_{1}-s\right)^{q-1}\right| \cdot\left[\varphi_{r}(s)+K^{*} \int_{0}^{s} \phi_{r}(\tau) d \tau\right] d s, \\
& I_{3}=\frac{M C_{\beta-\alpha}}{\Gamma(q)} \int_{t_{1}}^{t_{2}}\left(t_{2}-s\right)^{q-1} \cdot\left[\varphi_{r}(s)+K^{*} \int_{0}^{s} \phi_{r}(\tau) d \tau\right] d s .
\end{aligned}
$$

For any $\epsilon \in\left(0, t_{1}\right)$, we have

$$
\begin{aligned}
I_{1} \leq & C_{\beta-\alpha} \int_{0}^{t_{1}-\epsilon}\left(t_{2}-s\right)^{q-1}\left\|V\left(t_{2}-s\right)-V\left(t_{1}-s\right)\right\| \cdot\left[\varphi_{r}(s)+K^{* \prime} \int_{0}^{s} \phi_{r}(\tau) d \tau\right] d s \\
& +C_{\beta-\alpha} \int_{t_{1}-\epsilon}^{t_{1}}\left(t_{2}-s\right)^{q-1}\left\|V\left(t_{2}-s\right)-V\left(t_{1}-s\right)\right\| \cdot\left[\varphi_{r}(s)+K^{*} \int_{0}^{s} \phi_{r}(\tau) d \tau\right] d s \\
\leq & C_{\beta-\alpha} \int_{0}^{t_{1}-\epsilon}\left(t_{1}-s\right)^{q-1}\left[\varphi_{r}(s)+K^{\prime \prime} \int_{0}^{s} \phi_{r}(\tau) d \tau\right] d s \cdot \sup _{s \in\left[0, t_{1}-\epsilon\right]}\left\|V\left(t_{2}-s\right)-V\left(t_{1}-s\right)\right\| \\
& +\frac{2 M C_{\beta-\alpha}}{\Gamma(q)} \int_{t_{1}-\epsilon}^{t_{1}}\left(t_{1}-s\right)^{q-1} \cdot\left[\varphi_{r}(s)+K^{*} \int_{0}^{s} \phi_{r}(\tau) d \tau\right] d s .
\end{aligned}
$$

It follows from Lemma 3 that $I_{1} \rightarrow 0$ as $t_{2} \rightarrow t_{1}$ and $\epsilon \rightarrow 0$ independently of $u \in B_{r}$. From the expressions of $I_{2}$ and $I_{3}$, it is clear that $I_{2} \rightarrow 0$ and $I_{3} \rightarrow 0$ as $t_{2} \rightarrow t_{1}$ independently of $u \in B_{r}$. Therefore, we prove that $\left\{Q_{2} u: u \in B_{r}\right\}$ is a family of equicontinuous functions.

It remains to prove that for any $t \in J$, the set $W(t):=\left\{\left(Q_{2} u\right)(t): u \in B_{r}\right\}$ is relatively compact.

Obviously, $W(0)$ is relatively compact in $X_{\alpha}$. Let $0<t \leq T$ be fixed. For each $\delta \in(0, t)$, $\epsilon>0$ and $u \in B_{r}$, we define an operator $Q_{2}^{\delta, \epsilon}$ by

$$
\begin{aligned}
\left(Q_{2}^{\delta, \epsilon} u\right)(t)= & \int_{0}^{t-\delta}(t-s)^{q-1} \int_{\epsilon}^{\infty} q \theta \eta_{q}(\theta) S\left((t-s)^{q} \theta\right) f(s, u(s)) d \theta d s \\
& +\int_{0}^{t-\delta}(t-s)^{q-1} \int_{\epsilon}^{\infty} q \theta \eta_{q}(\theta) S\left((t-s)^{q} \theta\right) \int_{0}^{s} K(s, \tau) h(\tau, u(\tau)) d \tau d \theta d s \\
= & S\left(\delta^{q} \epsilon\right)\left[\int_{0}^{t-\delta}(t-s)^{q-1} \int_{\epsilon}^{\infty} q \theta \eta_{q}(\theta) S\left((t-s)^{q} \theta-\delta^{q} \epsilon\right) f(s, u(s)) d \theta d s\right.
\end{aligned}
$$




$$
\begin{aligned}
& +\int_{0}^{t-\delta}(t-s)^{q-1} \int_{\epsilon}^{\infty} q \theta \eta_{q}(\theta) S\left((t-s)^{q} \theta-\delta^{q} \epsilon\right) \\
& \left.\times \int_{0}^{s} K(s, \tau) h(\tau, u(\tau)) d \tau d \theta d s\right]
\end{aligned}
$$

Then the sets $W_{\delta, \epsilon}(t):=\left\{\left(Q_{2}^{\delta, \epsilon} u\right)(t): u \in B_{r}\right\}$ are relatively compact in $X_{\alpha}$ since by Lemma 2, the operator $S_{\alpha}(t)$ is compact for $t>0$ in $X_{\alpha}$. Moreover, for every $u \in B_{r}$, from Lemma 3 and assumption $\left(\mathrm{H}_{3}\right)$, we have

$$
\begin{aligned}
&\left\|\left(Q_{2} u\right)(t)-\left(Q_{2}^{\delta, \epsilon} u\right)(t)\right\|_{\alpha} \\
& \leq \| \int_{0}^{t}(t-s)^{q-1} \int_{0}^{\epsilon} q \theta \eta_{q}(\theta) S\left((t-s)^{q} \theta\right) \\
& \times\left[f(s, u(s))+\int_{0}^{s} K(s, \tau) h(\tau, u(\tau)) d \tau\right] d \theta d s \|_{\alpha} \\
&+\| \int_{t-\delta}^{t}(t-s)^{q-1} \int_{\epsilon}^{\infty} q \theta \eta_{q}(\theta) S\left((t-s)^{q} \theta\right) \\
& \quad \times\left[f(s, u(s))+\int_{0}^{s} K(s, \tau) h(\tau, u(\tau)) d \tau\right] d \theta d s \|_{\alpha} \\
& \leq \int_{0}^{t}(t-s)^{q-1}\left\|A^{\alpha-\beta} \int_{0}^{\epsilon} q \theta \eta_{q}(\theta) S\left((t-s)^{q} \theta\right) d \theta \cdot\left[\varphi_{r}(s)+K^{*} \int_{0}^{s} \phi_{r}(\tau) d \tau\right]\right\| d s \\
& \quad+\int_{t-\delta}^{t}(t-s)^{q-1}\left\|_{A^{\alpha-\beta}}^{\infty} \int_{\epsilon}^{\infty} q \theta \eta_{q}(\theta) S\left((t-s)^{q} \theta\right) d \theta \cdot\left[\varphi_{r}(s)+K^{*} \int_{0}^{s} \phi_{r}(\tau) d \tau\right]\right\| d s \\
& \leq q M C_{\beta-\alpha} \int_{0}^{t}(t-s)^{q-1}\left[\varphi_{r}(s)+K^{*} \int_{0}^{s} \phi_{r}(\tau) d \tau\right] d s \cdot \int_{0}^{\epsilon} \theta \eta_{q}(\theta) d \theta \\
&+\frac{M C_{\beta-\alpha}}{\Gamma(q)} \int_{t-\delta}^{t}(t-s)^{q-1}\left[\varphi_{r}(s)+K^{*} \int_{0}^{s} \phi_{r}(\tau) d \tau\right] d s .
\end{aligned}
$$

Therefore, there are relatively compact sets arbitrarily close to the set $W(t)$ for $t \in(0, T]$ and since it is compact at $t=0$, we have the relative compactness of $W(t)$ in $X_{\alpha}$ for all $t \in J$.

Therefore, the set $\left\{Q_{2} u: u \in B_{r}\right\}$ is relatively compact by the Ascoli-Arzela theorem. Thus, the continuity of $Q_{2}$ and relative compactness of the set $\left\{Q_{2} u: u \in B_{r}\right\}$ imply that $Q_{2}$ is a completely continuous operator. Hence, Krasnoselskii's fixed point theorem shows that the operator equation $u=Q_{1} u+Q_{2} u$ has a solution on $B_{r}$. Therefore, Eq. (1) has at least one mild solution. The proof is completed.

The following existence and uniqueness theorem for Eq. (1) is based on the Banach contraction principle. We will also need the following assumptions.

$\left(\mathrm{H}_{5}\right)$ There exists a constant $\beta \in[\alpha, 1]$ such that the functions $f, h: J \times X_{\alpha} \rightarrow X_{\beta}$ are strongly measurable.

$\left(\mathrm{H}_{6}\right)$ For $r>0$, there exist functions $\rho_{1}, \rho_{2} \in L^{1}\left(J, \mathbb{R}^{+}\right)$such that

$$
\begin{gathered}
\|f(t, x)-f(t, y)\|_{\beta} \leq \rho_{1}(t)\|x-y\|_{\alpha}, \\
\|h(t, x)-h(t, y)\|_{\beta} \leq \rho_{2}(t)\|x-y\|_{\alpha}
\end{gathered}
$$

for any $x, y \in B_{r}$ and $t \in J$. 
Theorem 2 Let the assumptions $\left(\mathrm{H}_{3}\right)-\left(\mathrm{H}_{6}\right)$ be satisfied. If $x_{0} \in X_{\alpha}$ and the inequalities (3) and

$$
\mu \triangleq M L+\frac{M T^{q} C_{\beta-\alpha}}{\Gamma(q+1)}\left(\left\|\rho_{1}\right\|_{L^{1}}+K^{*}\left\|\rho_{2}\right\|_{L^{1}}\right)<1
$$

hold, then Eq. (1) has a unique mild solution.

Proof From Lemma 4 and assumption $\left(\mathrm{H}_{5}\right)$, it is easy to see that $(t-s)^{q-1} V(t-s)[f(s, u(s))+$ $\left.\int_{0}^{s} K(s, \tau) h(\tau, u(\tau)) d \tau\right]$ is Bochner integrable with respect to $s \in[0, t]$ for all $t \in J$. For any $u \in B_{r}$, we define an operator $Q$ by

$$
\begin{aligned}
(Q u)(t)= & U(t)\left(x_{0}-g(u)\right)+\int_{0}^{t}(t-s)^{q-1} V(t-s) \\
& \times\left[f(s, u(s))+\int_{0}^{s} K(s, \tau) h(\tau, u(\tau)) d \tau\right] d s, \quad t \in J .
\end{aligned}
$$

According to the proof of Theorem 1, we know that $Q\left(B_{r}\right) \subset B_{r}$ for some $r>0$. For any $u, v \in B_{r}$ and $t \in J$, from Lemma 3, assumptions $\left(\mathrm{H}_{4}\right)$ and $\left(\mathrm{H}_{6}\right)$, we have

$$
\begin{aligned}
\|(Q u)(t)-(Q v)(t)\|_{\alpha} & \\
\leq & \|U(t)[g(v)-g(u)]\|_{\alpha} \\
& +\int_{0}^{t}(t-s)^{q-1}\|V(t-s)[f(s, u(s))-f(s, v(s))]\|_{\alpha} d s \\
& +\int_{0}^{t}(t-s)^{q-1}\left\|V(t-s) \int_{0}^{s} K(s, \tau)[h(\tau, u(\tau))-h(\tau, v(\tau))] d \tau\right\|_{\alpha} d s \\
\leq & M L\|u-v\|_{C}+\frac{q M C_{\beta-\alpha}}{\Gamma(q+1)} \int_{0}^{t}(t-s)^{q-1} \rho_{1}(s)\|u(s)-v(s)\|_{\alpha} d s \\
& +\frac{q M C_{\beta-\alpha} K^{*}}{\Gamma(q+1)} \int_{0}^{t}(t-s)^{q-1} \int_{0}^{s} \rho_{2}(\tau)\|u(\tau)-v(\tau)\|_{\alpha} d \tau d s \\
\leq & M L\|u-v\|_{C}+\frac{q M C_{\beta-\alpha}}{\Gamma(q+1)} \int_{0}^{t}(t-s)^{q-1} d s \cdot\left\|\rho_{1}\right\|_{L^{1}} \cdot\|u-v\|_{C} \\
& +\frac{q M C_{\beta-\alpha} K^{*}}{\Gamma(q+1)} \int_{0}^{t}(t-s)^{q-1} d s \cdot\left\|\rho_{2}\right\|_{L^{1}} \cdot\|u-v\|_{C} \\
= & {\left[M L+\frac{M T^{q} C_{\beta-\alpha}}{\Gamma(q+1)}\left(\left\|\rho_{1}\right\|_{L^{1}}+K^{*}\left\|\rho_{2}\right\|_{L^{1}}\right)\right] \cdot\|u-v\|_{C} . }
\end{aligned}
$$

Thus,

$$
\|Q u-Q v\|_{C} \leq \mu\|u-v\|_{C},
$$

which means that $Q$ is a contraction according to (4). By applying the Banach contraction principle, we know that $Q$ has a unique fixed point on $B_{r}$, which is the unique mild solution of Eq. (1). This completes the proof. 


\section{An example}

Let $X=L^{2}[0, \pi]$ equip with its natural norm $\|\cdot\|_{2}$ and inner product $\langle\cdot, \cdot\rangle$. Consider the following system:

$$
\left\{\begin{array}{l}
\frac{\partial^{\frac{1}{2}}}{\partial t^{\frac{1}{2}}} u(t, x)-\frac{\partial^{2}}{\partial x^{2}} u(t, x)=u(t, x) \sin t^{\frac{1}{2}}+\int_{0}^{t} K(t, s) u(s, x) \cos s d s, \\
\quad t \in[0, T], x \in[0, \pi], \\
u(t, 0)=u(t, \pi)=0, \quad t \in[0, T], \\
u(0, x)+\sum_{i=1}^{m} \int_{0}^{\pi} K_{0}(x, y) u\left(t_{i}, y\right) d y=u_{0}(x), \quad x \in[0, \pi],
\end{array}\right.
$$

where $T>0$ is a constant, $0<t_{1}<\cdots<t_{m}<T, u_{0} \in X$ and $K_{0}$ will be specified later.

Let the operator $A: D(A) \subset X \rightarrow X$ be defined by

$$
D(A):=\left\{v \in X: v^{\prime}, v^{\prime \prime} \in X, v(0)=v(\pi)=0\right\}, \quad A u=-\frac{\partial^{2} u}{\partial x^{2}} .
$$

Then $-A$ generates a compact analytic semigroup $S(\cdot)$ of uniformly bounded linear operators and $\|S(t)\|_{\mathcal{L}(X)} \leq e^{-t}$ for all $t \geq 0$. Hence, we take $M=1$. Moreover, the eigenvalues of $A$ are $n^{2}, n \in \mathbb{N}$ and the corresponding normalized eigenvectors are $z_{n}(x)=\sqrt{\frac{2}{\pi}} \sin (n x)$, $n=1,2, \ldots$. The operator $A^{\frac{1}{2}}$ is given by

$$
A^{\frac{1}{2}} \xi=\sum_{n=1}^{\infty} n\left\langle\xi, z_{n}\right\rangle z_{n}
$$

for each $\xi \in D\left(A^{\frac{1}{2}}\right):=\left\{v \in X: \sum_{n=1}^{\infty} n\left\langle v, z_{n}\right\rangle z_{n} \in X\right\}$ and $\left\|A^{-\frac{1}{2}}\right\|_{\mathcal{L}(X)}=1$.

Lemma 6 ([16]) If $\xi \in D\left(A^{\frac{1}{2}}\right)$, then $\xi$ is absolutely continuous, $\xi^{\prime} \in X$ and $\left\|\xi^{\prime}\right\|_{2}=\left\|A^{\frac{1}{2}} \xi\right\|_{2}$.

Let $X_{\frac{1}{2}}=\left(D\left(A^{\frac{1}{2}}\right),\|\cdot\|_{\frac{1}{2}}\right)$, where $\|\xi\|_{\frac{1}{2}}:=\left\|A^{\frac{1}{2}} \xi\right\|_{X}$ for all $\xi \in D\left(A^{\frac{1}{2}}\right)$. Assume that

$\left(\mathrm{P}_{1}\right)$ The function $K_{0} \in L^{2}([0, \pi] \times[0, \pi], \mathbb{R}), K_{0}(0, y)=K_{0}(\pi, y)=0$, and the partial derivative $(x, y) \mapsto \frac{\partial}{\partial x} K_{0}(x, y)$ belongs to $L^{2}([0, \pi] \times[0, \pi], \mathbb{R})$.

Define

$$
\begin{aligned}
& f(t, u(t))(x)=u(t, x) \sin t^{\frac{1}{2}}, \\
& h(t, u(t))(x)=u(t, x) \cos t \\
& g(u)(x)=\sum_{i=1}^{m} \int_{0}^{\pi} K_{0}(x, y) u\left(t_{i}, y\right) d y .
\end{aligned}
$$

Let $\xi \in X_{\frac{1}{2}}$, it follows from

$$
\begin{aligned}
\left\langle f(t, \xi), z_{n}\right\rangle & =\int_{0}^{\pi} u(t, x) \sin t^{\frac{1}{2}} \cdot \sqrt{\frac{2}{\pi}} \sin (n x) d x \\
& =\frac{1}{n} \int_{0}^{\pi}\left[\frac{\partial}{\partial x} u(t, x)\right] \sin t^{\frac{1}{2}} \cdot \sqrt{\frac{2}{\pi}} \cos (n x) d x
\end{aligned}
$$


and

$$
\begin{aligned}
\left\langle h(t, \xi), z_{n}\right\rangle & =\int_{0}^{\pi} u(t, x) \cos t \cdot \sqrt{\frac{2}{\pi}} \sin (n x) d x \\
& =\frac{1}{n} \int_{0}^{\pi}\left[\frac{\partial}{\partial x} u(t, x)\right] \cos t \cdot \sqrt{\frac{2}{\pi}} \cos (n x) d x
\end{aligned}
$$

that $f$ and $h$ are functions from $[0, T] \times X_{\frac{1}{2}}$ into $X_{\frac{1}{2}}$ and they are continuous. Moreover, a similar computation of [17] together with Lemma 6 and assumption $\left(\mathrm{P}_{1}\right)$ shows that $g(u) \in X_{\frac{1}{2}}$ whenever $u \in C\left([0, T], X_{\frac{1}{2}}\right)$.

Then for any $r>0$, we see that the assumptions $\left(\mathrm{H}_{3}\right)-\left(\mathrm{H}_{6}\right)$ are satisfied with

$$
\begin{aligned}
& \varphi_{r}(t)=r \sin t^{\frac{1}{2}}, \quad \phi_{r}(t)=r \cos t, \quad \sigma_{1}=\sigma_{2}=2 T^{\frac{1}{2}}, \\
& L=(n+1)\left(\int_{0}^{\pi} \int_{0}^{\pi}\left[\frac{\partial}{\partial x} K_{0}(x, y)\right]^{2} d x d y\right)^{\frac{1}{2}}, \quad \rho_{1}(t)=\rho_{2}(t)=1 .
\end{aligned}
$$

Thus, the system (5) has at least one mild solution due to Theorem 1 provided that $L+$ $\frac{2 T^{\frac{1}{2}}}{\sqrt{\pi}}\left(1+K^{*}\right)<1$. And by Theorem 2 , this mild solution of the system $(5)$ is unique on $[0, T] \times$ $[0, \pi]$.

\section{Competing interests}

The author declares that they have no competing interests.

\section{Acknowledgements}

Research was supported by the Fundamental Research Funds for the Gansu Universities.

Received: 8 August 2012 Accepted: 28 September 2012 Published: 17 October 2012

\section{References}

1. Gaul, L, Klein, P, Kempfle, S: Damping description involving fractional operators. Mech. Syst. Signal Process. 5, 81-88 (1991)

2. Glockle, WG, Nonnenmacher, TF: A fractional calculus approach of self-similar protein dynamics. Biophys. J. 68, 46-53 (1995)

3. Metzler, F, Schick, W, Kilian, HG, Nonnenmacher, TF: Relaxation in filled polymers: a fractional calculus approach. J. Chem. Phys. 103, 7180-7186 (1995)

4. Mainardi, F: Fractional calculus some basic problems in continuum and statistical mechanics. In: Carpinteri, A, Mainardi, F (eds.) Fractals and Fractional Calculus in Continuum Mechanics, pp. 291-348. Springer, Vienna (1997)

5. Hilfer, R: Applications of Fractional Calculus in Physics. World Scientific, Singapore (2000)

6. Zhou, Y, Jiao, F: Nonlocal Cauchy problem for fractional evolution equations. Nonlinear Anal. 11, $4465-4475$ (2010)

7. Wang, RN, Xiao, TJ, Liang, J: A note on the fractional Cauchy problems with nonlocal initial conditions. Appl. Math. Lett. 24, 1435-1442 (2011)

8. El-Borai, MM: Some probability densities and fundamental solutions of fractional evolution equations. Chaos Solitons Fractals 14, 433-440 (2002)

9. El-Borai, MM: Exact solutions for some nonlinear fractional parabolic partial differential equations. Appl. Math. Comput. 206, 150-153 (2008)

10. Wang, JR, Zhou, Y: Existence of mild solutions for fractional delay evolution systems. Appl. Math. Comput. 218, 357-367 (2011)

11. LV, ZW, Liang, J, Xiao, TJ: Solutions to the Cauchy problem for differential equations in Banach spaces with fractional order. Comput. Math. Appl. 62, 1303-1311 (2011)

12. Byszewski, L: Theorems about existence and uniqueness of solutions of a semi-linear evolution nonlocal Cauchy problem. J. Math. Anal. Appl. 162, 494-505 (1991)

13. Byszewski, L, Lakshmikantham, $\mathrm{V}$ : Theorems about the existence and uniqueness of a solution of a nonlocal abstract Cauchy problem in a Banach space. Appl. Anal. 40, 11-19 (1991)

14. Pazy, A: Semigroups of Linear Operators and Applications to Partial Differential Equations. Springer, New York (1983)

15. Liu, H, Chang, J: Existence for a class of partial differential equations with nonlocal conditions. Nonlinear Anal. 70 , 3076-3083 (2009)

16. Travis, CC, Webb, GF: Existence stability and compactness with $\alpha$-norm for partial functional differential equations. Trans. Am. Math. Soc. 240, 129-143 (1978)

17. Chang, J, Liu, H: Existence of solutions for a class of neutral partial differential equations with nonlocal conditions in the $\alpha$-norm. Nonlinear Anal. 71, 3759-3768 (2009) 
doi:10.1186/1687-2770-2012-113

Cite this article as: Yang: Existence of mild solutions for fractional evolution equations with nonlocal conditions. Boundary Value Problems 2012 2012:113.

Submit your manuscript to a SpringerOpen ${ }^{\circ}$ journal and benefit from:

- Convenient online submission

- Rigorous peer review

- Immediate publication on acceptance

- Open access: articles freely available online

- High visibility within the field

- Retaining the copyright to your article

Submit your next manuscript at $\gg$ springeropen.com 